\title{
APUNTES SOBRE LA COOPERACIÓN INTERNACIONAL PARA EL DESARROLLO Y LA RESPONSABILIDAD SOCIAL FRENTE A LOS OBJETIVOS DE DESARROLLO DEL MILENIO Y EL PACTO GLOBAL
}

IBELIS BLANCO RANGEL*

Recibido 24 de Mayo de 2011/Enviado para Modificación 9 de Octubre de 2011/Aceptado 30 de Noviembre de 2011

\begin{abstract}
RESUMEN
El presente artículo, es una revisión sobre la relación de la Cooperación Internacional para el Desarrollo y la Responsabilidad Social teniendo como elemento central la relación entre los Objetivos de Desarrollo del Milenio, y el Pacto Global. Se encuentra dividido en dos partes: La primera, describe la Cooperación internacional para el desarrollo así como el enfoque de desarrollo al que se hará referencia, seguidamente se argumenta los roles que ha desempeñado el sector privado, como beneficiaria de cooperación y como financiadora de desarrollo. La segunda parte, puntualiza el concepto de la Responsabilidad Social que se tomará como referencia, posteriormente se hará de la relación entre los Objetivos de Desarrollo del Milenio y el Pacto Global.
\end{abstract}

Palabras Clave: Cooperación Internacional, Desarrollo, Responsabilidad Social, Objetivos de Desarrollo del Milenio, Pacto Global.

Clasificación JEL: O11, O15, R11, R58, 019

\footnotetext{
* Socióloga de la Universidad Católica Andrés Bello (Venezuela) cursó estudios de postgrado en Gerencia de Proyectos de Investigación y Desarrollo en la Universidad Central de Venezuela, es Especialista en Cooperación Internacional para el Desarrollo de la Universidad San Buenaventura (Colombia) en convenio con la Universidad de Pavía (Italia). Ha desarrollado su experiencia profesional en investigación social y evaluación de proyectos sociales. Participó en la coordinación del VI Conferencia Internacional del Banco Interamericano de Desarrollo. Actualmente es co-investigadora de la línea financiamiento al desarrollo del grupo de investigación de la Escuela Latinoamericana de Cooperación y Desarrollo. Correo Electrónico: ibelis.blanco@gmail.com
} 


\begin{abstract}
This article reviews the relationship of the International Cooperation for Development and Social Responsibility having as central element the relationship between the Millennium Development Goals and the Global Compact. It is divided into two parts: The first describes the international development cooperation and development approach to which reference will be made, then the argument roles played by the private sector as a beneficiary of cooperation and development as Funding. The second part, explains the concept of social responsibility to be taken as a reference, then made of the relationship between the Millennium Development Goals and the Global Compact.
\end{abstract}

Key Words: International Cooperation, Development, Social Responsibility, Millennium Development Goals, the Global Compact.

JEL Classification: O11, O15, R11, R58, 019

\title{
LA COOPERACIÓN INTERNACIONAL PARA EL DESARROLLO
}

Para introducir la relación entre la cooperación para el desarrollo y la responsabilidad social de las empresas, es necesario ubicar el contexto de cada para entender los puntos en los que se relacionan.

Por lo cual se partió de la pregunta ¿qué es la Cooperación Internacional? Un de las clásicas respuestas, la describe como un instrumento de las relaciones internacionales, fruto del "sistema institucional de la modernidad" (Guiddens, 1994) ha sido objeto de estudio de las ciencias políticas, razón por la cual privilegia a los estados nacionales como principales actores de cooperación.

En el siglo XX, surgió la Cooperación Internacional para el Desarrollo como respuesta para superar los efectos producidos por la Segunda Guerra Mundial. El primer gran ejemplo el plan para la reconstrucción de Europa, mejor conocido como Plan Marshall.

Por lo cual una de las diferencias entre la Cooperación Internacional y la Cooperación Internacional para el Desarrollo es que en la primera participan los estados nacionales, mientras que en la segunda, además de éstos también participan organismos internacionales, sociedad civil y otras instituciones estatales, lo cual genera otras dinámicas de orden internacional. 
Como se hablará de cooperación para el desarrollo, es necesario precisar a qué tipo de desarrollo se hará referencia. En este caso se asumirán los enfoques de desarrollo propuestos por:

- La Comisión Mundial sobre Medio Ambiente y Desarrollo,

- El Programa de Naciones Unidas para el Desarrollo (PNUD) de Naciones Unidas y Banco Mundial (1).

El primer enfoque, se refiere al desarrollo sostenible término utilizado en 1987 por la Comisión Mundial sobre Medio Ambiente y Desarrollo que en su informe Brudtland hace mención al desarrollo como aquel que "satisface las necesidades de la generación actual sin comprometer la capacidad de las generaciones futuras para satisfacer sus propias necesidades".

El segundo enfoque, propuesto en los años noventa por el PNUD introduce el término Desarrollo Humano en los Informes de Desarrollo Humano $^{1}$ donde se considera a la persona como el centro del desarrollo. El desarrollo humano es definido como "un proceso mediante el cual se amplían las oportunidades de los individuos" (2) señalando como oportunidades de mayor prioridad: una vida prolongada y saludable, acceso a la educación y el disfrute de un nivel de vida decente.

Al finalizar el siglo $X X$ se fueron agregando otros elementos en torno al desarrollo, hasta llegar al Desarrollo integral introducido por el Banco Mundial, el cual propone trabajar el desarrollo macroeconómico y el socio-estructural en conjunto, siendo necesario cuatro condiciones para un desarrollo integrado.-Según Wolfensohn (3), esas condiciones son:

- Estructurales; (gobierno, sistemas jurídico, financiero y de seguridad);

- humanas, (salud, educación)

- materiales (agua, saneamiento, energía, transporte y comunicaciones, problemas ambientales y culturales) y

- estrategias del sector privado.

Precisamente bajo esta perspectiva de desarrollo, es evidente la entrada en vigor del sector privado (representado por la gran empresa) como uno de los responsables de los procesos de desarrollo.

\footnotetext{
${ }^{1}$ Son documentos independientes elaborado por un equipo de académicos y profesionales del desarrollo del PNUD. El objetivo del informe es situar a la persona en el centro del proceso de desarrollo, no se limita al nivel de ingreso sino que tiene que ver con las opciones y libertades que tiene cada persona.
} 
Si a lo anterior se le suma que en los últimos años, las modalidades y enfoques de la Cooperación Internacional para el Desarrollo han sido objeto de crítica y reflexión, orientado a que ésta "ha alcanzado sólo avances aislados no logrando contribuir sustancial e integralmente al desarrollo y disminución de la pobreza en los países en vías de desarrollo" (4), con mayor razón se necesita reconocer que ni los estados ni la sociedad civil son suficientes para apalancar los procesos de desarrollo.

\section{PAPEL DE LA EMPRESA EN LA COOPERACIÓN PARA EL DESARROLLO}

Por el rol social y económico que tiene la empresa, no es usual ubicarla en el escenario de cooperación parecería. Sin embargo en los años noventa, el Banco mundial introduce el "enfoque multiparticipativo" ${ }^{2}(5)$ en los procesos de desarrollo, lo cual propició la entrada de actores no tradicionales en la CID, como los gobiernos locales, la sociedad civil y desde inicio del siglo XXI.

Este enfoque surge a partir de los procesos democráticos (6), el cual tomó mayor fuerza con la Declaración del Milenio (en 2000) de las que se derivaron los Objetivos de Desarrollo del Milenio ${ }^{3}(7)$.

Los procesos de globalización económica, también favorecieron la entrada del sector privado en algunos temas de interés público, tales como: los procesos de integración regional y subregional, los flujos financieros internacionales (de carácter lucrativo y no lucrativo) y los avances en las ciencias de información y comunicación.

Luego de una exhaustiva revisión de literatura, sobre los actores de cooperación, fue escasa la información sobre la participación del sector privado. En todo caso, el hallazgo encontrado fue que las grandes empresas de los países como Noruega y Holanda (8) han estado relacionadas en los procesos de cooperación para desarrollo desde los años sesenta.

Si bien se ha hecho referencia a la gran empresa, es necesario aclarar que las Pequeñas y Medianas Empresas (en adelante Pymes) especialmente europeas, han recibido beneficios de cooperación internacional. Por lo

\footnotetext{
${ }^{2}$ También conocido como multista kehorlder.

${ }^{3}$ Los ODM son ocho: Erradicar la pobreza extrema y el hambre, educación universal, igualdad entre los géneros, reducir la mortalidad infantil, mejorar la salud materna, combatir el VIH/SIDA, Sostenibilidad el medio ambiente, Fomentar una asociación mundial
} 
que se deduce que dependiendo de su tamaño, la empresa ha jugado dos roles dentro de la cooperación para el desarrollo. En un primer momento como beneficiaria y posteriormente como financiadora.

\section{La empresa como beneficiaria de cooperación}

Esta modalidad tiene la finalidad de ofrecer incentivos para fortalecer a las Pymes, ha sido promovida por los estados europeos y se encuentra dentro de la cooperación económica. En España recibe el nombre de Cooperación al Desarrollo con Participación Empresarial (en adelante CDPE) y según Guzmán y Rama puede ser entendida por:

Los estímulos brindados por el Estado del país cooperante o por los organismos supranacionales a la participación de la empresa de un país industrializado. En coinversiones, transferencias de tecnología, estudios de factibilidad, formación profesional y otros servicios desempeñados con o para empresas ... de un país en desarrollo (8).

Ello significa, que el estado le interesa potenciar el sector productivo teniendo en cuenta las ventajas comparativas que puede resultar de las relaciones económicas y comerciales que se establecen dentro de los acuerdos macro de colaboración entre países. Además, que éstas se puede dar entre países de renta alta y los países de renta media o baja e incluso entre países de renta media bajo la perspectiva de la cooperación sur - sur.

Vale la pena resaltar, que estos acuerdos se realizan bajo una serie de criterios de solidaridad y cooperación que deben estar por encima de los intereses de las empresas y están acompañados de un grado de condicionalidad con el objetivo de fomentar la capacidad empresarial.

Otro punto importante, es la rentabilidad constante que debe estar presente tanto para la empresa del país industrializado como para la empresa receptora, ello no quiere decir que excluya el componente cooperación más bien se dice que tiene un carácter mixto. Las formas que derivan este tipo de cooperación van desde concesiones de licencias para comercializar o producir un producto, hasta la creación conjunta de empresas o Joint-Venture.

Un ejemplo de Joint-venture o cooperación empresarial (norte-sur), es el caso de la empresa Española Casual Brand que en conjunto con la empresa colombiana Procafecol, que en 2007 se plantearon abrir 40 tiendas Juan 
Valdez y en tres años que además de café, venden artículos artesanales 100\% Colombianos para apoyar a una asociación sin ánimo de lucro situada en el Departamento de Santander (9).

$\mathrm{Al}$ igual que las otras modalidades de cooperación, el sector productivo en Europa cuenta con una serie de instrumentos para acceder a la cooperación. Desde 1993 se encuentra disponible en América Latina uno de esos instrumentos: el Banco Europeo de Inversiones que en la actualidad mantiene vínculos con Argentina, Brasil, Chile y Uruguay a través de la transferencia de tecnología y la financiación de proyecto enfocados en temas de medio ambiente y energía.

El marco europeo ofrece otro aparte al respecto, está relacionado con los programas de cooperación al sector empresarial mejor conocido como Alinvest que desde 1995 apoyana la internacionalización de las pymes para contribuir a la cohesión social.

En el caso de América Latina el organismo del Banco Interamericano de Desarrollo (BID) que fomenta la inversión de las Pymes es la Corporación Interamericana de Inversiones (CII).

De lo anterior podemos hacer las siguientes consideraciones:

- Los organismos tanto gubernamentales como multilaterales tiene claro que al fomentar el sector empresarial apalancan procesos de desarrollo (no sólo económico)

- Existen programas e instrumentos institucionalizados para apoyar al sector empresarial de las Pymes.

- Cooperar no es lo mismo que ayudar, lleva implícito beneficios tanto para quien recibe como para quien otorga la cooperación, es decir, donde todos deberían ganar, ello lo hace atractivo dentro de la lógica mercantil.

a. La empresa como financiadora de desarrollo

Como financiadora de desarrollo, la gran empresa, comienza a ser vista como actor emergente de cooperación al desarrollo en el contexto internacional. 
Entre los argumentos que propicia este nuevo rol se encuentran:

- El enfoque multicipativo (antes mencionados)

- La cumbre de Río de 1992 donde se pone en la agenda, la importancia de entrar iniciar el tema delen diálogo con las empresas poren los temas relacionados con el medio ambiente.

La iniciativa del Pacto Global ${ }^{4}$ (10) propuesta por Naciones Unidas en 1999 en Suiza con el objetivo de que las empresas multinacionales y transnacionales de manera voluntaria"alineen sus estrategias" de negocio de manera voluntaria enfocándolas en cuatro grandes áreas temáticas: derechos humanos, estándares laborales, medio ambiente y anticorrupción.

\section{PRINCIPIOS DEL PACTO GLOBAL}

\section{DERECHOS HUMANOS}

Principio 1: Las empresas deben apoyar y respetar la protección de los derechos humanos fundamentales, reconocidos internacionalmente, dentro de su ámbito de influencia

Principio 2: Las empresas deben asegurarse de que sus empresas no son cómplices en la vulneración de los derechos humanos

\section{ESTANDARES LABORALES}

Principio 3: Las empresas deben apoyar la libertad de asociación y el reconocimiento efectivo del derecho a la negociación colectiva

Principio 4: Las empresas deben apoyar la eliminación de toda forma de trabajo forzoso o realizado bajo coacción

Principio 5: Las Empresas deben apoyar la erradicación del trabajo infantil. Principio 6: Las Empresas deben apoyar la abolición de las prácticas de discriminación en el empleo y ocupación.

\section{MEDIO AMBIENTE}

Principio 7: Las Empresas deberán mantener un enfoque preventivo que favorezca el medio ambiente.

\footnotetext{
${ }^{4}$ Es importante resaltar que este documento retoma principios de: La declaración de los Derechos Humanos, la Declaración de Principios de la Organización Internacional del Trabajo relativa a los derechos fundamentales en el trabajo, la Declaración de Río sobre el Medio Ambiente y el Desarrollo, y desde 2004, la Convención de la Naciones Unidas contra la Corrupción
} 
Principio 8: Las empresas deben fomentar las iniciativas que promuevan una mayor responsabilidad ambiental.

Principio 9: Las Empresas deben favorecer el desarrollo y la difusión de las tecnologías respetuosas con el medio ambiente

\section{ANTICORRUPCION}

Principio 10: Las Empresas deben trabajar en contra de la corrupción en todas sus formas, incluidas la extorsión y el soborno.

Si bien en el Pacto las empresas comunican los progresos de sus acciones en los cuatro principios, también participan organizaciones de la sociedad civil y academia.

En América Latina existen varias oficinas del Pacto Global desde México hasta Argentina. El Centro Regional para América Latina y el Caribe y funciona en Bogotá - Colombia.

Otro argumento de gran importancia para argumentar que la empresa es un actor de cooperación, es la valoración que le han dado organismos internacionales como el Banco Mundial sobre el potencial que puede tenerde la empresa al servicio de los menos favorecidos. Tal es el caso de concepto "la fortuna en la base de la pirámide", del profesor C.K Prahalad quequien parte del supuesto de que los si bien existen cuatro billones de personas que viven con menos de 2 dólares al día pueden ser vistos como un mercado potencial si las empresas generan estrategias para que ellos puedan acceder a los productos que ofrecen.

\section{LA RESPONSABILIDAD SOCIAL}

Hablar de Responsabilidad Social Empresarial como generalmente se conoce, es limitar esta herramienta de las ciencias administrativas a las empresas bien sea multinacionales transnacionales o pequeñas y medianas. Pero sería inadecuado la utilización de éste término porque hoy día se reconoce que ésta esta abarca todo tipo de organizaciones, por ello se hablará de Responsabilidad Social

Teniendo presente, que desde 2000 la Organización Internacional para la Estandarización comenzó un proceso de diagnóstico y discusión sobre la Responsabilidad que terminó en septiembre de 2010con la publicación de la guía sobre Responsabilidad Social, se toma como referencia el concepto que ellos proponen ya que está legitimado a nivel internacional 
"Responsabilidad de una organización ante los impactos que sus decisiones y actividades ocasionan en la sociedad y el medio ambiente mediante un comportamiento ético y transparente que contribuya al desarrollo sostenible, incluyendo la salud y el bienestar de la sociedad; tome en consideración las expectativas de sus partes interesadas; cumpla con la legislación aplicable y sea coherente con la normativa internacional de comportamiento; y esté integrada en toda la organización y se lleve a la práctica en sus relaciones" (11).

Esta definición subraya:

- La importancia de la sociedad en general y de las partes interesadas en particular, entendida esta como grupo de personas o instituciones que se relacionan con la organización,

- El medio ambiente y las consecuencias que pueden recaer sobre él cuando no se toman en cuenta algunos factores.

- La ética y transparencia en favor del desarrollo sostenible.

Relación entre Cooperación para el Desarrollo y Responsabilidad Social Se dice que la cooperación internacional es a las relaciones internacionales lo que la responsabilidad social es a las ciencias administrativas, pero además tienen varios puntos en común. Uno de ellos es que forman parte de las ciencias sociales, pero además, el punto que las hace convergentes es que ambas son modalidades cuyo fin último es contribuir con el desarrollo sostenible.

Hoy día los actores de cooperación, se relacionan con el sector privado y a su vez el sector privado está buscando acercarse a las diferentes partes interesadas que han trabajado en temas social y medio ambiental para hacer sinergia y generar mecanismos de colaboración de mutuo beneficio. Así lo afirmó en 2010 la secretaria de Estado de Cooperación Internacional de España Soraya Rodríguez Ramos "tenemos que asociar al sector privado en nuestras actuaciones".

\section{OBJETIVOS DE DESARROLLO DEL MILENIO Y PACTO GLOBAL}

A principios del milenio, se abrió una nueva perspectiva para quienes trabajan en temas de Desarrollo gracias a la adopción de los Objetivos de Desarrollo del Milenio (ODM) que fueron concebido a raíz de la cumbre del Milenio celebrada en Nueva York en septiembre de 2000 cuando 189 naciones reconocieron que uno de los valores fundamentales para las 
relaciones internacionales del siglo XXI es la responsabilidad común de la "gestión del desarrollo económico y social en el mundo" (Declaración del Milenio, 2000).

Después su declaración Tiempo después, los Objetivos han sido analizados y revisados en distintos consensos como Monterrey (2002) y Roma (2003) respectivamente y luego durante el segundo foro de Alto Nivel sobre la eficacia de la ayuda celebrado en Paris (2005) lo cual, propuso una nueva orientación sobre cómo la Cooperación Internacional para el Desarrollo sobre cuáles son los grandes temas que se debe hacer frente, ellos son:

\section{OBJETIVOS DE DESARROLLO DEL MILENIO}

Objetivo 1. Erradicar la pobreza extrema y el hambre

Objetivo 2. Lograr la enseñanza primaria universal

Objetivo 3. Promover la igualdad de género y el empoderamiento de la mujer

Objetivo 4. Reducir la mortalidad de los niños menores de 5 años

Objetivo 5. Mejorar la salud materna

Objetivo 6. Combatir el VIH/SIDA, el paludismo y otras enfermedades

Objetivo 7. Garantizar la sostenibilidad del medio ambiente

Objetivo 8. Fomentar una alianza mundial para el desarrollo

Una de las características de los ODM es que son considerados una guía debido a que cuenta con metas e indicadores para medir los avances o retrocesos que se han producido en a partir de 2000 en materia de Desarrollo. Precisamente en esta nueva orientación hace énfasis en la importancia del actor emergente en escenario de la cooperación internacional para el desarrollo: las empresas.

A continuación se presentan algunos puntos de coincidencia entre Ahora bien, en cuanto a su relaciónlos Objetivos de Desarrollo del Milenio (7) ycon el Pacto Global (10), es posible encontrar algunas coincidencias.

La primera es que ambas, son propuestas por el mismo organismo: Naciones Unidas. La segunda, es que los principios del Pacto Global están fueron formulados en concordancia con los Objetivos de Desarrollo del Milenio. los cuales son fruto donde los países miembros reconocieron que uno de los valores fundamentales para las relaciones internacionales del siglo XXI es la responsabilidad común de la "gestión del desarrollo 
económico y social en el mundo" (Declaración del Milenio, 2Además, si se revisa en detalle las metas propuestas de los objetivos de los ODM es posible encontrar la relación con uno de los principios del Pacto Global. Por ejemplo la meta 1.B con estándares laborales.

- "Lograr empleo pleno y productivo, y trabajo decente para todos, incluyendo mujeres y jóvenes" y el indicador relacionado con; (meta 1 B de los ODM)

- "Proporción de escaños ocupados por mujeres en los parlamentos nacionales "tiene que ver con la categoría Estándares laborales, en especial con el principio 6 Las Empresas deben apoyar la abolición de las prácticas de discriminación en el empleo y ocupación.

De igual forma, los principios contenidos sobre el Medio ambiente del Pacto Global esta en armonía con los indicadores del Objetivo 7: Garantizar la sostenibilidad del Medio Ambiente que se presentan a continuación:

- Proporción de la superficie cubierta por bosques

- Emisiones de dióxido de carbono (total, per cápita y por cada dólar PPA del PIB)

- Proporción del total de recursos hídricos utilizada

- Proporción de las áreas terrestres y marinas protegidas

- Proporción de especies en peligro de extinción

- Proporción de población con acceso a fuentes mejoradas de abastecimiento de agua potable

- Proporción de población con acceso a servicios de saneamiento mejorados

Finalmente, el principio relacionado con la ética y la transparencia, es decir con la ANTICORRUPCIÓN tiene relación con las metas 8.E y 8.F del Objetivo 8 de los ODM Fomentar una alianza mundial para el desarrollo. La meta $8 \mathrm{~F}$ plantea que la alianza para el desarrollo se bebe realizar en colaboración con el sector privado para brindar acceso a la información y comunicaciones. Ello se debe a los beneficios de las Tecnologías de Información y Comunicación. (TIC).

En tanto, la meta 8E está dirigida a una industria específica: la farmacéutica y tiene como propósito "proporcionar acceso a medicamentos esenciales en los países en desarrollo a precios asequibles" 


\section{CONCLUSIONES}

De la revisión de la literatura sobre el tema es posible deducir algunas conclusiones:

- La empresa es un actor emergente en la cooperación internacional como financiador de procesos de desarrollo. Este rol ha sido promovido (en parte) por organismos internacionales.

- La cooperación internacional y la responsabilidad social tiene como punto en común el desarrollo sostenible.

- Las empresas deben ser vistas como aliadas estratégicas del desarrollo.

- No se debe reducir el término de Responsabilidad Social a las empresas sino mas bien considerarlo para todo tipo de organizaciones que estén dispuestas a tomar en cuenta factores sociales y ambientales.

\section{REFERENCIAS BIBLIOGRÁFICAS}

1. Naciones Unidas. Documentación de las Naciones Unidas - Guía de Investigación. [En línea] [Citado el: 20 de Enero de 2010.] http:// www.un.org/depts/dhl/spanish/resguids/specenvsp.htm.

2. Programa de Naciones Unidas Para el Desarrollo. Informes Sobre Desarrollo Humano. Programa de Naciones Unidas Para el Desarrollo - Informes Sobre Desarrollo Humano. [En línea] [Citado el: 6 de Octubre de 2010.] http:/ / hdr.undp.org/es/informes/.

3. División de Desarrollo Sostenible y Asentamientos Humanos CEPAL. La ciudad inclusiva. Santiago de Chile: Naciones Unidas, 2003. 92-1-322259-9.

4. Bonfiglio, Giovanni. Nuevas Tendencias de la Cooperación Internacional y análisis de un caso en Perú. Universidad San Martín de Porres - Instituto del Perú. [En línea] Diciembre de 2009. [Citado el: 20 de Enero de 2010.] http://institutodelperu.org.pe/descargas/ nuevas_tendencias_de_la_coop_int.pdf.

5. Banco Mundial. Banco Mundial. Enfoquese iniciativas de desarrollo del Banco Mundial. [En línea] [Citado el: 2010 de Febrero de 2010.] http:/ / web.worldbank.org/WBSITE/EXTERNAL/BANCOMUNDIAL/ EXTTEMAS/EXTCSOSPANISH/0,,contentMDK:20625039 menuPK :1614096 pagePK:220503 piPK:220476 theSitePK:1490924,00.html.

6. Castaño, Teba y Larrú,José María. Nuevas herramientas decooperación al desarrollo ¿Nuevo rol de los actores? Madrid: CYAN. PROYECTOS Y PRODUCCIONES EDITORIALES, S.L., 2005. 9788481986174.

7. Organización de las Naciones Unidas. Objetivos de Desarrollo del 
Milenio. Organización de Naciones Unidas. [En línea] [Citado el: 10 de Agosto de 2010.] http://www.un.org/spanish/millenniumgoals/.

8. Andrés López, Gonzalo y Gonzalo Molina de la Torre, Ignacio. INTRODUCCION A LA SOLIDARIDAD INTERNACIONAL. LA COOPERACIÓN PARA EL DESARROLLO. Valladolid : Universidad de Valladolid - Secretariado de Publiaciones e Intercambio Editorial, 2000. 978-84-8448-059-4.

9. Europa Press. Economía/Empresas.- Casual Brands y Procafecol crean una 'joint venture' para abrir 40 tiendas Juan Valdez en tres años. elEconomista.es. [En línea] 18 de Junio de 2007. [Citado el: 5 de Noviembre de 2010.] http:/ / ecodiario.eleconomista.es/empresasfinanzas/noticias / 231105/06/07/Economia-Empresas-CasualBrands-y-Procafecol-crean-una-joint-venture-para-abrir-40-tiendasJuan-Valdez-en-tres-anos-.html.

10. Organización de las Naciones Unidas. Principios del Pacto Global. Organización de las Naciones Unidas. [En línea] [Citado el: 10 de Agosto de 2010.] http://www.ungloblcompact.org/Languages/ spanish/Los_Diez_Principios.html.

11. ISO (International Organization for Standardization). ISO - 26000 Social Responsibility. International Organization for Standardization. [En línea] 27 de Junio de 2010. [Citado el: 15 de Octubre de 2010.] http://www.iso.org/iso/iso_26000_project_overview.pdf.

\section{BIBLIOGRAFÍA RECOMENDADA}

Blanco, I (2010). Responsabilidad Social y Cooperación para el Desarrollo. La empresa como actor de cooperación. Revista de Negocios \& Gerencia. Universidad Católica Andrés Bello, 2.

Buchelli. J. (2008).La Cooperación descentralizada: ¿un escenario común entre las relaciones internacionales y el desarrollo?Ponencia presentada en primero Congreso de Ciencias políticas de la Universidad de los Andes, Octubre, Bogotá.

Comisión Económica para América Latina y el Caribe. Recuperado el 9 de febrero de 2010 http://www.eclac.org/cgi-bin/getProd.asp?xml=/ $\mathrm{mdg} /$ noticias/paginas/6/35556/P35556.xml\&xsl=/mdg/tpl/p18f-st. xsl\&base $=/ \mathrm{mdg} / \mathrm{tpl} /$ top-bottom.xsl.

Dinero, (s.f).Futuroqueestállegando. Recuperadoel 20 deoctubre de2010.http:/ / www.dinero.com/wf_ImprimirArticulo.aspx?IdRef=16070\&Id Tab=1. 
Giddens, A. (1994).Consecuencias de la modernidad. Madrid: Alianza.

Gómez, M y Sanahuja, J. (1999). El sistema internacional de cooperación al desarrollo, una aproximación a sus actores e instrumentos. Madrid: CIDEAL.

Gómez, M y Sanahuja, J. (2001). La cooperación al desarrollo en un mundo de cambio: perspectivas sobre nuevos ámbitos de intervención. Madrid: CIDEAL.

Julio, My Nivia-Ruiz, F. (2009). Cooperación Euroandina y sur-sur en América Latina y Caribe. Cartagena de Indias: San Buenaventura-Cartagena.

Olcese, A. Rodríguez, M. Alfaro, J. (2008).Manual de la empresa responsable y sostenible. Conceptos, ejemplos y herramientas de la Responsabilidad Social Corporativa o de la Empresa.Madrid; Mc Graw Hill.

Prado, J (2005).La dimensión ética de la cooperación internacional al desarrollo, Entre la solidaridad y el poder en las relaciones internacionales. Recuperado el 09 de octubre de 2009, de http:/ / www.iadb.org/Etica/Documentos/ pra_ladim.pdf.

The World Bank Group.(s.f.).Stakeholder Analysis.Recuperado el 15 de octubre de 2010 de http://www1.worldbank.org/publicsector/ anticorrupt/PoliticalEconomy/stakeholderanalysis.htm.

"Colabora empresa, que luego es tarde", El País Negocios, 27 de junio de 2010. 